\title{
LIE-POISSON STRUCTURE ON SOME POISSON LIE GROUPS
}

\author{
VIKTOR L. GINZBURG AND ALAN WEINSTEIN
}

\section{INTRODUCTION}

Poisson Lie groups appeared in the work of Drinfel' d (see, e.g., [Dr1, Dr2]) as classical objects corresponding to quantum groups. Going in the other direction, we may say that a Poisson Lie group is a group of symmetries of a phase space that are allowed to "twist," in a certain sense, the symplectic or Poisson structure. The Poisson structure on the group controls this twisting in a precise way. Quantizing both the phase space and the symmetries, one may obtain a quantum group acting on a quantum phase space.

In recent work, Lu and Ratiu [LR] used so-called standard Poisson structures on a compact semisimple Lie group $K$ and on its Poisson dual $K^{*}$ in order to give a new proof of the nonlinear convexity theorem of Kostant [Ko]. Their method is analogous to the famous symplectic proof of the linear convexity theorem given by Atiyah [A] and Guillemin and Sternberg [GS]. The nonlinear convexity theorem, like the linear one, follows from a very general result on convexity of the image of the momentum map [A, GS]; however, in [LR] it is applied not to a coadjoint orbit in $\mathfrak{k}^{*}$, but to a symplectic leaf in $K^{*}$.

The main result of this paper is that the standard Poisson structure on the Poisson dual $K^{*}$ to a compact semisimple Poisson Lie group $K$ is actually isomorphic to the linear one on $\mathfrak{k}^{*}$. This theorem seems to be related to some facts in the theory of quantum groups. Namely, (for generic $q$ ) the universal enveloping algebra $U(\mathfrak{k})$ and its quantum deformation $U_{q}(\mathfrak{k})$ are isomorphic as algebras (though not as coalgebras, of course, since the quantum version is not cocommutative). In particular, there is a bijective correspondence between their representations. A direct connection between our work and its quantum analogues, though, is still to be found.

The present work supplies a positive answer to Question 5.1 in [LR] and strongly depends on that paper. To simplify reading, we keep the notations of [LR] wherever possible, on one hand, but give all necessary definitions, on the other.

Our work is also related to [Du], in which the nonlinear convexity theorem is reduced to the linear one by a deformation argument not unlike the one that we use in $\S 5$.

The paper is organized as follows. In $\S 2$ we define Poisson Lie groups, discuss

Received by the editors July 30,1991 .

1991 Mathematics Subject Classification. Primary 58F05; Secondary 17B56.

The research was partially supported by NSF Grants DMS-85-05550 and DMS-90-01089. 
some of their properties, and describe the standard Poisson structures on a compact semisimple Lie group and on its Poisson dual. More detailed information on these Poisson Lie groups may be found in [LW].

Sections 3 and 4 are devoted to preliminary results needed for the proof of our main theorem. In particular, we prove in $\S 3$ that if a compact Lie group $G$ acts on a manifold $M$, then the cohomology of the Lie algebra $\mathfrak{g}$ with coefficients in $C^{\infty}(M)$ is equal to the tensor product of $H^{\star}(\mathfrak{g})$ and the ring Inv of $G$-invariant smooth functions on $M$. Thus, $C^{\infty}(M)$ behaves like a finite-dimensional $\mathfrak{g}$-module. (This result follows from the work of van Est [E], but we have chosen to give our proof since it is so short and essentially selfcontained.) In particular, $H^{2}\left(\mathfrak{g}, C^{\infty}(M)\right)=0$ if $G$ is semisimple. The section is independent of [LR] and of $\S 2$.

The last section is entirely devoted to the proof of our main result.

\section{Poisson LIE GROUPS}

Here we briefly discuss the notion of Poisson Lie group and give all necessary definitions. The proofs of results we mention may be found, for example, in [LW].

Definition 2.1. A Poisson Lie group $(G, \pi)$ is a Lie group equipped with a Poisson structure $\pi$ such that the multiplication $G \times G \rightarrow G$ is a Poisson map, where $G \times G$ carries the product Poisson structure.

It is easy to show that the Poisson structure $\pi$ must vanish at the identity $e \in G$, so that its linearization $d_{e} \pi: \mathfrak{g} \rightarrow \mathfrak{g} \wedge \mathfrak{g}$ at $e$ is well defined. (Here $\mathfrak{g}$ is the Lie algebra of $G$.) This linear homomorphism turns out to be a 1-cocycle with respect to the adjoint action, and the dual homomorphism $\mathfrak{g}^{*} \wedge \mathfrak{g}^{*} \rightarrow \mathfrak{g}^{*}$ satisfies the Jacobi identity; i.e., $\mathfrak{g}^{*}$ becomes a Lie algebra.

The preceding construction is in some sense invertible. Namely, assume that we are given Lie algebra structures on $\mathfrak{g}$ and $\mathfrak{g}^{*}$ and that the homomorphism $\mathfrak{g} \rightarrow \mathfrak{g} \wedge \mathfrak{g}$ dual to the bracket on $\mathfrak{g}^{*}$ is a cocycle. Then the homomorphism $\mathfrak{g}^{*} \rightarrow \mathfrak{g}^{*} \wedge \mathfrak{g}^{*}$ dual to the bracket on $\mathfrak{g}$ is a cocycle as well, and the simply connected Lie groups $G$ and $G^{*}$ with the Lie algebras $\mathfrak{g}$ and $\mathfrak{g}^{*}$, respectively, carry uniquely defined Poisson structures $\pi$ and $\pi^{*}$, making them into Poisson Lie groups for which the underlying linearizations are the original structures. The Poisson Lie groups $(G, \pi)$ and $\left(G^{*}, \pi^{*}\right)$ are said to be dual to each other.

The Lie algebra $\mathfrak{g}$ acts on the manifold $G^{*}$; i.e., we have a Lie algebra homomorphism from $\mathfrak{g}$ to the Lie algebra of vector fields on $G^{*}$. To describe this action, we pick an element $v \in \mathfrak{g}=\left(\mathfrak{g}^{*}\right)^{*}$. It may be identified with an element $\alpha_{e} \in T^{*} G^{*}$. Let us extend $\alpha_{e}$ to a right invariant 1-form $\alpha$ on $G^{*}$. Then the vector field $v_{*}$ corresponding to $v$ is obtained from $-\alpha$ by means of the Poisson structure $\pi^{*}$. One may check that in this way we get an action of $\mathfrak{g}$ on $G^{*}[\mathrm{~W}]$. Hence, if all the vector fields $v_{*}$ are complete, we can integrate the $\mathfrak{g}$-action and obtain a $G$-action on $G^{*}$ called the dressing action.

Trivial example. Let $G$ be any Lie group equipped with the zero Poisson structure. Then $G^{*}=\mathfrak{g}^{*}$ with its natural Poisson and (additive) Lie group structures. The dressing action is just the coadjoint action. 
In general the dressing action preserves neither the group structure nor the Poisson structure on $G^{*}$. Nevertheless, it is Poisson in the sense that the map $G \times G^{*} \rightarrow G^{*}$ is a Poisson map. Moreover, the symplectic leaves of $\pi^{*}$ are precisely the orbits of the $G$-action.

In the rest of this section, we are going to describe our main object, the socalled standard Poisson structures on compact connected semisimple Lie groups and their duals. First we change our notation slightly to match that in [LR].

From now on, let $G^{\mathbf{C}}$ be a complex semisimple Lie group and $K$ its compact real form.

Example. The standard Poisson Lie structures on $K$ and $K^{*}$. Fix an Iwasawa decomposition $G^{\mathbf{R}}=K A N$ (see [H]) of $G^{\mathbf{C}}$ regarded as a real group $G^{\mathbf{R}}$, and let $<,>$ be the imaginary part of the complex Killing form on $G^{\mathrm{C}}$. Denote the Lie algebras of $G^{\mathbf{R}}, K$, and $B=A N$ by $\mathfrak{g}^{\mathbf{R}}, \mathfrak{k}$, and $\mathfrak{b}$, respectively. One can check that $\mathfrak{k}$ and $\mathfrak{b}$ are isotropic subspaces of $\mathfrak{g}^{\mathbf{R}}$ with respect to $<,>$. Therefore, since $<,>$ is nondegenerate, $\mathfrak{b}$ is identified with $\mathfrak{k}^{*}$. The cocycle condition is satisfied, and thus both $K$ and $B=K^{*}$ become Poisson Lie groups.

Example. $G^{\mathrm{C}}=\mathrm{SL}(n, \mathrm{C}), K=\mathrm{SU}(n)$; then $B$ is the group $\mathrm{SB}(n, \mathrm{C})$ of all $n \times n$ upper triangular complex matrices with determinant one and real positive elements on the diagonal. The Poisson Lie group structure on $\mathrm{SU}(n)$ was described by $\mathrm{Lu}$ and Weinstein in [LW]. We prove below that the Poisson structures on $\operatorname{su}(n)^{*}$ and $\mathrm{SB}(n, \mathrm{C})$ are diffeomorphic.

Remark. Since the group $K$ is compact and semisimple, it follows from the result of Conn [C] that, in a neighborhood of $e \in K^{*}$, the Poisson structure $\pi^{*}$ is diffeomorphic to the standard one on a neighborhood of the origin in $\mathfrak{k}^{*}$.

Theorem 2.2. The Poisson structures on $\mathfrak{k}^{*}$ and $K^{*}$ are globally diffeomorphic.

Remark. The Lie groups $\mathfrak{k}^{*}$ and $K^{*}$ are obviously not isomorphic. Therefore, $\mathfrak{k}^{*}$ and $K^{*}$ cannot be isomorphic as Poisson Lie groups.

\section{Poisson cohomology AND Lie Algebra COHOMOLOGY}

Let $\Omega_{k}$ be the space of all $k$-vector fields on a Poisson manifold $(P, \pi)$. Recall that the graded vector space $\Omega_{\star}$ carries a natural bilinear homomorphism [ , ]: $\Omega_{k} \times \Omega_{l} \rightarrow \Omega_{k+l-1}$ called the Schouten bracket and satisfying the graded Jacobi identity (see, e.g., $[\mathrm{Ku}]$ ). As a consequence, the linear operator $d_{\pi}: \Omega_{k} \rightarrow$ $\Omega_{k+1}, d_{\pi} w=[\pi, w]$ is, in fact, a differential: $d_{\pi}^{2}=0$.

Definition 3.1. The Poisson cohomology $H_{\pi}^{\star}(P)$ of $(P, \pi)$ is the cohomology of the complex $\left(\Omega_{\star}, d_{\pi}\right)$.

Example. If $(P, \omega)$ is a symplectic manifold then $H_{\pi}^{\star}(P)=H^{\star}(P)$. In fact, we have the isomorphism of the complex $\left(\Omega_{\star}, d_{\pi}\right)$ and the de Rham complex of $P$ given by the formula $v_{1} \wedge \cdots \wedge v_{k} \mapsto i_{v_{1}} \omega \wedge \cdots \wedge i_{v_{k}} \omega$.

Let us now come back to our main objects: a Poisson Lie group $G$ and its dual group $G^{*}$. The goal of this section is to calculate the Poisson cohomology 
of $G^{*}$ in terms of the Lie algebra cohomology of $\mathfrak{g}$. Denote by Inv the algebra of all Casimir functions on $G^{*}$, i.e., functions constant on the leaves of the symplectic foliation. Equivalently, Inv is the algebra of $G$-invariant functions with respect to the dressing action of $G$ on $G^{*}$.

Theorem 3.2. If $G$ is a compact Poisson Lie group then $H_{\pi}^{\star}\left(G^{*}\right)=H^{\star}(\mathfrak{g}) \otimes \operatorname{Inv}$.

Corollary 3.3. If $G$ is a compact semisimple Poisson Lie group then $H_{\pi}^{2}\left(G^{*}\right)=0$.

Remark. We do not require $G$ to be equipped with the standard Poisson structure.

We will not need this theorem in its full generality. In fact, all that we need is the corollary. However, the proof in this particular case does not differ too much from the general one, which we prefer.

Theorem 3.2 follows from the next two results.

Proposition 3.4 [L]. If $G$ is any Poisson Lie group then

$$
H_{\pi}^{\star}\left(G^{*}\right)=H^{\star}\left(\mathfrak{g}, C^{\infty}\left(G^{*}\right)\right),
$$

where $C^{\infty}\left(G^{*}\right)$ is made into a $\mathfrak{g}$-module by means of the dressing action of $\mathfrak{g}$ on $G^{*}$.

The proof of this proposition is rather standard (see, e.g., [L] or [GL]) and we omit it. Note also that a similar result holds for any open subset $U \subset G^{*}$. Namely, $H_{\pi}^{\star}(U)=H^{\star}\left(\mathfrak{g}, C^{\infty}(U)\right)$.

The right-hand member of the equality in Proposition 3.4 may be calculated in an even more general situation. Consider a compact group $G$ acting on a (not necessary compact) manifold $M$, and denote by Inv the algebra of $G$-invariant smooth functions on $M$.

Theorem 3.5. $H^{\star}\left(\mathfrak{g}, C^{\infty}(M)\right)=H^{\star}(\mathfrak{g}) \otimes \operatorname{Inv}$.

Proof of Theorem 3.2. Combine Theorem 3.5 and Proposition 3.4. Q.E.D.

Proof of Theorem 3.5. Consider the direct product $G \times M$ with two commuting $G$-actions $T^{r}$ and $T^{l}, T^{r}(g)(h, x)=\left(h g^{-1}, g x\right)$ and $T^{l}(g)(h, x)=$ $(g h, x)$, where $g, h \in G, x \in M$, and $x \mapsto g x$ is just the action of $g$ on $M$. Orbits of these actions are precisely fibers of the fibrations $\rho^{r}, \rho^{l}: G \times M \rightarrow M$, where $\rho^{r}(h, x)=h x$ and $\rho^{l}$ is just the natural projection $(h, x) \mapsto x$. Let $\Omega^{\star}\left(\rho^{r}\right)$ be the complex of $T^{l}$-invariant differential forms on the $\rho^{r}$-fibers equipped with the de Rham differential $d$.

Denote by $\mathscr{C}^{\star}$ the Chevalley-Eilenberg complex for the cohomology of $\mathfrak{g}$ with coefficients in $C^{\infty}(M)$.

Lemma 3.6. There exists a natural isomorphism of complexes $\Phi: \mathscr{C}^{\star} \rightarrow \Omega^{\star}$.

Proof of Lemma 3.6. To define $\Phi$ pick $c \in \mathscr{C}^{k}$ and vectors $v_{1}, \ldots, v_{k}$ tangent to the $T^{r}$-orbit at a point $(g, x) \in G \times M$. There are $k$ uniquely defined elements $a_{1}, \ldots, a_{k}$ in $\mathfrak{g}$ such that for the corresponding vector fields $T_{*}^{r}\left(a_{i}\right)$, $i=1, \ldots, k$ on $G \times M$ we have $T_{*}^{r}\left(a_{i}\right)_{(g, x)}=v_{i}$. Let us set

$$
\Phi c_{(g, x)}\left(v_{1}, \ldots, v_{k}\right)=c\left(a_{1}, \ldots, a_{k}\right)(x) \text {. }
$$


Since the actions $T^{l}$ and $T^{r}$ commute, the $k$-form $\Phi_{c}$ is $T^{l}$-invariant.

The linear homomorphism $\Phi$ is, in fact, an isomorphism. Indeed, let $\sigma \in$ $\Omega^{k}\left(\rho^{r}\right)$ and $a_{1}, \ldots, a_{k} \in \mathfrak{g} ;$ then

$$
\Phi^{-1} \sigma\left(a_{1}, \ldots, a_{k}\right)(x)=\sigma\left(T_{*}^{r} a_{1}, \ldots, T_{*}^{r} a_{k}\right)(g, x)
$$

is well defined since $\sigma$ and all of the $T_{*}^{r}\left(a_{i}\right)$ are $T^{l}$-invariant.

A straightforward calculation shows that $\Phi$ is actually an isomorphism of complexes. Q.E.D.

By Lemma 3.6 we see that

$$
H^{\star}\left(\Omega^{\star}\left(\rho^{r}\right), d\right)=H^{\star}\left(\mathfrak{g}, C^{\infty}(M)\right),
$$

and it remains to show that

$$
H^{\star}\left(\Omega^{\star}\left(\rho^{r}\right), d\right)=H^{\star}(\mathfrak{g}) \otimes \operatorname{Inv} .
$$

It is slightly simpler to work with the complex $\Omega^{\star}\left(\rho^{l}\right)$ formed by $T^{r}$-invariant forms on the $\rho^{l}$-fibers, which is isomorphic to $\Omega^{\star}\left(\rho^{r}\right)$ via the involution $(h, x)$ $\mapsto\left(h^{-1}, h x\right)$ of $G \times M$ that switches the actions $T^{l}$ and $T^{r}$.

Fix a $T^{r}$-invariant metric on $G \times M$, and let $\mathscr{H}^{\star}$ be the linear space of fiberwise harmonic forms in $\Omega^{\star}\left(\rho^{l}\right)$. The $\left(T^{r}\right.$-invariant) fiberwise Hodge decomposition implies that $H^{\star}\left(\Omega^{\star}\left(\rho^{l}\right), d\right)=\mathscr{H}^{\star}$. On the other hand, $\mathscr{H}^{\star}$ coincides with the linear space of all $G$-invariant smooth functions on $M$ with values in $H^{\star}(G)=H^{\star}(\mathfrak{g})$. Therefore,

$$
H^{\star}\left(\Omega^{\star}\left(\rho^{r}\right), d\right)=H^{\star}\left(\Omega^{\star}\left(\rho^{l}\right), d\right)=\mathscr{H}^{\star}=H^{\star}(\mathfrak{g}) \otimes \text { Inv. Q.E.D. }
$$

Remarks. 1. Our proof shows that there exists an operator $K$, from the space of all exact cochains $c \in \mathscr{C}^{\star}$ to $\mathscr{C}^{\star-1}$, such that $d_{\text {Lie }} K c=c$, where $d_{\text {Lie }}$ is the differential in the Chevalley-Eilenberg complex; this operator preserves smooth dependence on parameters.

2. We felt that Theorem 3.5 should be in the literature somewhere. After asking many people about such a reference, we learned from $\mathrm{D}$. Wigner that the theorem follows easily from Theorem 2 of [E]. On the other hand, the proof above is simple and self-contained except for the use of Hodge theory.

For bounded domains in Euclidean space, a result very close to Theorem 3.5 was also proved by Conn [C], who also found bounds for the operator $K$ mentioned in the previous remark. Lemma 3.6 is not quite new either. A similar result was proved by Weinstein and $\mathrm{Xu}[\mathrm{WX}]$ in the much more general context of groupoids, and the same method was used in [GL] in order to prove the Morita invariance of the first Poisson cohomology.

3. The only simplification that would occur in a direct proof of Corollary 3.3 would be to notice that $H^{2}(G)=0$ if $G$ is semisimple and to prove that $H^{2}\left(\Omega^{\star}\left(\rho^{l}\right), d\right)=0$ using again the fiberwise Hodge theory.

4. Theorem 3.5 does not extend to the case where one simply has a Lie algebra action. For instance, consider the coadjoint action of $\mathrm{SU}(2)$ restricted to the complement of a line through the origin. The resulting action is defined only at the level of the Lie algebra, and the resulting cohomology is not trivial in 
dimension 1. One may see this by identifying the Lie algebra cohomology with Poisson cohomology and observing that the doubly-punctured spheres admit locally hamiltonian vector fields that are not globally hamiltonian.

\section{RIGIDITY OF INVARIANT SYMPLECTIC STRUCTURES}

In this section, we establish a sort of rigidity of symplectic structures invariant with respect to a given torus action and satisfying certain extra conditions.

Consider a compact manifold $W$ equipped with an action of a torus $T$. Suppose that the set $W^{T}$ of fixed points is discrete.

Proposition 4.1. If $\omega_{0}$ and $\omega_{1}$ are two $T$-invariant symplectic structures on $W$ that admit momentum maps $J_{0}$ and $J_{1}$, respectively, such that $J_{0}(x)=J_{1}(x)$ for every $x \in W^{T}$, then the cohomology classes of the symplectic structures coincide, $\left[\omega_{0}\right]=\left[\omega_{1}\right]$.

Remarks. 1. If we are given a family of $T$-invariant symplectic structures $\omega_{t}$, $t \in[0,1]$ with the momentum maps $J_{t}$ such that both $\omega_{t}$ and $J_{t}$ are smooth in $t$ and $J_{t}(x)$ is independent of $t$ for every $x \in W^{T}$, then the proposition and (equivariant) Moser's theorem [M] imply that all $\left(W, \omega_{t}\right)$ are equivariantly diffeomorphic.

2. If $\operatorname{dim} T=\operatorname{dim} W / 2$, no continuous deformation is necessary. Namely, it was proved by Delzant [D] that the image of the momentum map determines the symplectic structure up to an equivariant diffeomorphism.

3. The hypothesis that the fixed points of the $T$-action are isolated may be weakened and replaced by the assumption that the cohomology classes of $\omega_{0} \mid W^{T}$ and $\omega_{1} \mid W^{T}$ coincide.

Proof. Throughout the proof all cohomology groups will be taken with real coefficients.

First assume that $T$ is a circle and identify the dual space $t^{*}$ with $\mathbf{R}$. Let $\xi$ be a vector field generating the $T$-action. In other words, $\xi$ is a hamiltonian vector field for both the symplectic structures $\omega_{0}$ and $\omega_{1}$ with the hamiltonians $J_{0}$ and $J_{1}$, respectively.

Following Atiyah and Bott $[\mathrm{AB}]$, we recall that the $T$-equivariant de Rham complex of $W$ is the graded algebra $\Omega_{T}^{\star}[u]$ of polynomials in $u, \operatorname{deg} u=2$, with the coefficients in $T$-invariant differential forms, equipped with the differential $d_{T}=d+i_{\xi} u$. The cohomology of the complex $\left(\Omega_{T}^{\star}[u], d_{T}\right)$ coincides with the equivariant cohomology $H_{T}^{\star}(W)$ of $W$ [AB]. It is known (see [K]) that the restriction homomorphism

$$
j_{W^{T}}^{*}: H_{T}^{\star}(W) \rightarrow H_{T}^{\star}\left(W^{T}\right)=H^{\star}\left(W^{T}\right) \otimes \mathbf{R}[u]
$$

is injective because the $T$-action is hamiltonian with respect to either symplectic structure.

In particular, consider the elements $\omega_{0}^{T}=\omega_{0}+J_{0} u$ and $\omega_{1}^{T}=\omega_{1}+J_{1} u$ of the equivariant de Rham complex. These elements are $d_{T}$-closed and the restriction homomorphism $j_{W^{T}}^{*}$ sends their cohomology classes into $\bigoplus_{x \in W^{T}} J_{0}(x) u$ and $\bigoplus_{x \in W^{T}} J_{1}(x) u$, respectively. Since $j_{W^{T}}^{*}$ is injective and $J_{0}\left|W^{T}=J_{1}\right| W^{T}$, we 
see that $\left[\omega_{0}^{T}\right]=\left[\omega_{1}^{T}\right]$ in $H_{T}^{2}(W)$. Since the cohomology classes $\left[\omega_{0}\right]$ and $\left[\omega_{1}\right]$ are just the images of $\left[\omega_{0}^{T}\right]$ and $\left[\omega_{1}^{T}\right]$ under the natural homomorphism $H_{T}^{\star}(W) \rightarrow H^{\star}(W)$ "forgetting" the $T$-action, $\left[\omega_{0}\right]=\left[\omega_{1}\right]$.

The general case where $\operatorname{dim} T>1$ can either be proved in the same manner as the one above or reduced to it as follows. Let $\operatorname{dim} T>1$; recall that only a finite number of subgroups of $T$ may appear as stabilizers of points of $W$ (see, e.g., [B]). Therefore, there exists a one-dimensional closed subgroup $T^{1} \subset T$ that meets the stabilizer of every point in $W \backslash W^{T}$ along a discrete subgroup and thus $W^{T^{1}}=W^{T}$. It is obvious that both of the momentum maps of the $T^{1}$ action coincide on $W^{T^{1}}$. This means that the action of the circle $T^{1}$ satisfies the hypothesis of the proposition. Q.E.D.

\section{Proof OF THEOREM 2.2}

With all preparations in place, we can now prove Theorem 2.2.

Recall that $K$ is a compact semisimple Poisson Lie group equipped with the standard Poisson structure, $K^{*}$ is its dual. Let $\pi$ be the Poisson structure on $K^{*}$; except for this insignificant modification we will keep all the notation of $\S 2$. There exists a natural diffeomorphism $E: \mathfrak{k}^{*} \rightarrow K^{*}$ (see [LR, §5]), and hence we may identify (the manifold) $K^{*}$ with the linear space $\mathfrak{k}^{*}$.

Although it is not really necessary for our purpose we prefer to recall the definition of $E$. The subspaces $\mathfrak{k}$ and $\mathfrak{p}=i \mathfrak{k} \subset \mathfrak{g}^{\mathbf{R}}$ are isotropic with respect to the imaginary part $<,>$ of the complex Killing form. Therefore, $\mathfrak{p}$ may be identified with $\mathfrak{k}^{*}$ by means of $<,>$. The exponential map is, in fact, a diffeomorphism between $\mathfrak{p}$ and $P=\exp \mathfrak{p}$. The Cartan decomposition $G^{\mathbf{R}}=K P$ (see, e.g., $[\mathrm{H}]$ ), along with the Iwasawa decomposition $G^{\mathbf{R}}=K B$, where $B=K^{*}$, gives rise to a diffeomorphism $P \rightarrow K^{*}$. Combining all these diffeomorphisms we obtain $E$.

Now consider the family $\pi_{t}, t \in[0,1]$ of Poisson structures on $K^{*}=$ $\mathfrak{k}^{*}$ defined by the formula $\pi_{t}(x)=\pi(t x) / t$. Then $\pi_{1}=\pi$, and $\pi_{0}$ is the linearization of $\pi$ at the origin, i.e., the standard Lie-Poisson structure on $\mathfrak{k}^{*}$.

According to [LR], $E$ sends coadjoint orbits to orbits of the dressing action, so the symplectic foliations of $\pi$ and $\pi_{0}$ coincide. Since the foliation for $\pi_{0}$ is invariant under homotheties, all the $\pi_{t}$ have the same symplectic foliation as well. In other words, all of the $\pi_{t}$ are tangent to the coadjoint orbits in $\mathfrak{k}^{*}$ and symplectic on them. We need the following

Lemma 5.1. On every coadjoint orbit $\mathscr{O} \subset \mathfrak{k}^{*}$, all the symplectic structures $\omega_{t}$ corresponding to the Poisson structures $\pi_{t}$ lie in the same cohomology class.

Proof. Although $E$ is not Poisson, it is $T$-equivariant (and even $K$-equivariant), where $T \subset K$ is the maximal torus used in the Iwasawa decomposition. Since the $T$-action on $\mathfrak{k}^{*}$ preserves the Poisson structure $\pi$ [LR], it preserves all the $\pi_{t}$. Let $J_{t}: \mathscr{O} \rightarrow \mathfrak{t}$ be the momentum map of the $T$-action with respect to the symplectic structure $\omega_{t}$. The fixed points of the $T$-action are precisely those where the rank of $J_{t}$ is equal to zero. As was proved in [LR], the momentum maps $J_{0}$ and $J_{1}$ may be chosen to have the same image: $J_{0}(\mathscr{O})=J_{1}(\mathscr{O})$; 
moreover, $J_{0}(x)=J_{1}(x)$ for all the fixed points $x$ of the $T$-action. This implies that all the $J_{t}$ may be chosen so that $J_{t}(x)=$ const for every fixed point $x$. Hence the hypothesis of Proposition 4.1 holds for $W=\mathscr{O}$ and the lemma follows. Q.E.D.

To prove Theorem 2.2, we consider the bivector field $\dot{\pi}_{t}=d \pi_{t} / d t$ and, in particular, $\dot{\pi}=\dot{\pi}_{1}$. We claim that there exists a vector field $X$ such that

(1) $\dot{\pi}=L_{X} \pi$

(2) $X$ has the zero linearization at the origin;

(3) $X$ is tangent to the coadjoint orbits in $\mathfrak{k}^{*}$.

Obviously, $[\dot{\pi}, \pi]=0$, i.e., $\dot{\pi}$ is a Poisson cocycle on $\left(\mathfrak{k}^{*}, \pi\right)$. By Corollary 3.3, $\dot{\pi}$ is a Poisson coboundary; i.e., there exists a vector field $Y$ such that $\dot{\pi}=[\pi, Y]\left(=L_{Y} \pi\right)$.

All the Poisson structures $\pi_{t}$ vanish only at the origin and have the same linearization $\pi_{0}$ there. This implies that $Y$ vanishes at the origin and that its linearization $d_{0} Y$ is Poisson with respect to $\pi_{0}$, i.e., $L_{d_{0} Y} \pi_{0}=0$. Thus $L_{d_{0} Y}$ is dual to a derivation of $\mathfrak{g}$ that, since $\mathfrak{g}$ is semisimple, must be inner. Hence there is a linear function $f$ on $\mathfrak{g}^{*}$ such that $d_{0} Y$ is precisely the hamiltonian vector field for the hamiltonian $f$ with respect to the Poisson structure $\pi_{0}$. Finally, let $\xi$ be the hamiltonian vector field determined by $f$, but this time with respect to the Poisson structure $\pi$. The linearization of $\xi$ is the same as that of $Y$, and $X=Y-\xi$ satisfies both (1) and (2).

To prove (3), note first that $\dot{\pi}$ is tangent to the orbits, so the flow of $X$ must preserve the singular foliation by the coadjoint orbits. Lemma 5.1 implies that the flow of $X$ sends every generic coadjoint orbit onto an orbit with the same cohomology class $\left[\omega_{1}\right]=\left[\omega_{0}\right]$. Since the algebra $\mathfrak{k}$ is semisimple, the cohomology class $\left[\omega_{0}\right]$ varies nontrivially in all transversal directions to a generic orbit. Therefore, the flow preserves a generic orbit, i.e., $X$ is tangent to the generic orbits of the coadjoint action.

Consider now the time dependent vector field $X_{t}$ given by the formula $X_{t}(x)=X(t x) / t^{2}$. By (2), the field $X_{t}$ is well defined for $t=0$ and smooth in $t \in[0,1]$. By (3), all the $X_{t}$ are tangent to the generic coadjoint orbits. It follows that they are tangent to the (compact) level surfaces of the quadratic Casimir function given by the Killing form, so the family $X_{t}$ can be globally integrated to a family $\phi_{t}$ of diffeomorphisms. Finally, a straightforward calculation based on (1) shows that $L_{X_{t}} \pi_{t}=\dot{\pi}_{t}$. Therefore, $\phi_{t *} \pi_{0}=\pi_{t}$ and, in particular, $\phi_{1}$ is the desired diffeomorphism $\phi_{1 *} \pi_{0}=\pi$. This finishes the proof of Theorem 2.2. Q.E.D.

\section{ACKNOWLEDGMENTS}

We would like to thank J.-H. Lu and T. Ratiu for useful discussions and explanations concerning the nonlinear convexity theorem. We also wish to thank David Wigner for providing the reference to the paper of van Est cited in $\S 3$. It is impossible to mention all the other mathematicians who made suggestions to us regarding the proof of Theorem 3.5. We are deeply grateful to all of them. Finally, the second author would like to thank the Collège de France and the 
Universite Paris VI for their hospitality during the period when this manuscript was completed.

\section{REFERENCES}

[A] M. F. Atiyah, Convexity and commuting Hamiltonians, Bull. London Math. Soc. 14 (1982), $1-15$.

[ABo] M. Atiyah and R. Bott, The moment map and equivariant cohomology, Topology 23 (1984), 1-28.

[B] G. Bredon, Introduction to compact transformation groups, Pure and Appl. Math. vol. 46, Academic Press, New York, 1972.

[C] J. Conn, Normal forms for smooth Poisson structures, Ann. of Math. (2) 121 (1985), 565593.

[D] T. Delzant, Hamiltoniens périodiques et images convexes de l'application moment, Bull. Soc. Math. France 116 (1988), 315-339.

[Dr1] V. G. Drinfel'd, Hamiltonian structures on Lie groups, Lie bialgebras and the geometric meaning of the classical Yang-Baxter equations, Soviet Math. Dokl. 27 (1983), 68-71.

[Dr2] __, Quantum groups, Proc. ICM, Berkeley 1 (1986), 789-820.

[Du] J. J. Duistermaat, On the similarity between the Iwasawa projection and the diagonal part, Société Mathématique de France, 2e série, Mémoire no. 15, 1984, pp. 129-138.

[E] E. T. van Est, Une application d'une méthode de Cartan-Leary, Indag. Math. 17 (1955), 542-544.

[F] D. B. Fuks, Cohomology of infinite-dimensional Lie algebras, Consultants Bureau, New York, 1986.

[GL] V. L. Ginzburg and J. -H. Lu, Poisson calculus: Vector fields and differential forms (in preparation).

[GS] V. Guillemin and S. Sternberg, Convexity properties of the moment mapping, Invent. Math. 67 (1982), 491-513.

[H] S. Helgason, Differential geometry, Lie groups, and symmetric spaces, Academic Press, New York, 1978.

[K] F. Kirwan, Cohomology of quotients in symplectic and algebraic geometry, Math. Notes, vol. 31, Princeton Univ. Press, Princeton, NJ, 1984.

[Ko] B. Kostant, On convexity, the Weyl group and the Iwasawa decomposition, Ann. Sci. Ecole Norm. Sup. (4) 6 (1973), 413-455.

[Ku] J. L. Koszul, Crochet de Schouten-Nijenhuis et cohomologie, Élie Cartan et les Mathématiques d'Aujourd'hui, Astérisque hors série (1985), 257-271.

[L] J.-H. Lu, Multiplicative and affine Poisson structures on Lie groups, Berkeley Thesis, 1990.

[LR] J.-H. Lu and T. Ratiu, On the nonlinear convexity theorem of Kostant, J. Amer. Math. Soc. 4 (1991), 349-363.

[LW] J.-H. Lu and A. Weinstein, Poisson Lie groups, dressing transformations, and Bruhat decompositions, J. Differential Geom. 31 (1990), 501-526.

[M] J. Moser, On the volume elements on a manifold, Trans. Amer. Math. Soc. 120 (1965), 280-296.

[W] A. Weinstein, Some remarks on dressing transformations, J. Fac. Sci. Univ. Tokyo, Sect. A, Math. 36 (1988), 163-167.

[WX] A. Weinstein and P. Xu, Extensions of symplectic groupoids and quantization, J. Reine Angew. Math. 417 (1991), 159-189.

Mathematical Sciences Research Institute, Berkeley, California 94720

Current address: Department of Mathematics, Stanford University, Stanford, California 94305

Department of Mathematics, University of California, Berkeley, California 94720 